\title{
Men's Sheds as Community-based Health Promotion for Men Aged 50 Plus (MASH): Protocol for a Mixed-methods Systematic Review
}

Birte Albrecht ( $\sim$ b.albrecht@uni-bremen.de )

University of Bremen: Universitat Bremen https://orcid.org/0000-0002-1072-6767

Linda Foettinger

University of Bremen: Universitat Bremen

Karin Bammann

University of Bremen: Universitat Bremen

Protocol

Keywords: Men's sheds, self-rated health, subjective well-being, social isolation

Posted Date: December 4th, 2020

DOI: https://doi.org/10.21203/rs.3.rs-118163/v1

License: @ (1) This work is licensed under a Creative Commons Attribution 4.0 International License. Read Full License

Version of Record: A version of this preprint was published at Systematic Reviews on August 4th, 2021. See the published version at https://doi.org/10.1186/s13643-021-01762-x. 


\section{Abstract}

Background Men are exposed to different health risks than women. For instance, older men have an increased risk of social isolation. At the same time, they are less likely to participate in health promotion interventions and there is a lack of men-specific interventions. Men's sheds are a concept of community-based health promotion for older men where they can engage in joint activities. Prior research revealed various health-related effects of men's sheds, such as reduced social isolation and improved psychological well-being. Yet, only two reviews conducted in 2013 are available which both found an insufficient evidence base. Since the wider implementation of men's sheds is a recent phenomenon, and most of the literature on men's sheds has been published after 2013, a mixed-methods systematic review will be conducted to strengthen the current evidence base of men's sheds by analysing their effectiveness regarding self-rated health, subjective well-being, and social isolation. Moreover, information on how to successfully implement men's sheds will be gathered.

Methods This mixed-methods systematic review will follow the guidelines of The Joanna Briggs Institute (JBI). The databases Medline (via PubMed), Scopus, Web of Science, OpenGrey, and the websites of men's sheds associations will be searched for publications. Additionally, a hand-search in the reference lists of the included publications will be conducted. Qualitative and quantitative studies published in English, German, or French will be considered for inclusion. The quality of the selected studies will be assessed using the JBI critical appraisal checklists. Following the convergent segregated approach, the data synthesis will be undertaken independently, and subsequently combined in a mixed-methods data synthesis.

Discussion The results of this systematic review will lead to a comprehensive understanding of the current evidence base regarding the effectiveness of men's sheds. Furthermore, they will provide useful implications for the implementation of men's sheds.

Systematic review registration The systematic review is registered at the International Prospective Register of Systematic Reviews (PROSPERO; registration number forthcoming).

\section{Background}

In most countries, men significantly differ from women in terms of life expectancy, morbidity, and health-related behaviour [1]. In Germany, the life expectancy of men at birth, for instance, is five years lower than that of women [2]. Gender differences can also be observed in participation in health promotion and prevention programs. Although men are exposed to greater health risks than women, they are less likely to participate in such interventions [3]. This has been attributed to a low level of acceptance as well as the absence of male-oriented communication strategies to adequately address this specific target group [3-5]. Additionally, there is a lack of men-specific interventions to promote health [6].

Men's sheds are a concept of community-based health promotion for older men, originally developed in Australia. A men's shed is a non-profit communal institution for men where they can engage in joint activities. They are predominantly set up as easy-toaccess public organisations. The range of activities and general conditions differ from shed to shed. However, repairs and woodwork are central, especially for social and charitable purposes [6]. The Australian Men's Shed Association (AMSA) has defined improving well-being and health as major aims of men's sheds [7].

Previous studies found that older men have an increased risk of social isolation [8]. Due to its negative effects on mental and physical health, interventions should focus on strengthening the social relationships and integration [9]. Since men's sheds provide various opportunities to socialise and therefore decrease the risk of social isolation, they can make a substantial contribution to existing health promotion concepts $[10,11]$. The shoulder-to-shoulder communication in a supportive environment also enables men to talk about sensitive and shameful topics, including diseases or symptoms [10]. Thus, men's sheds are a valuable intervention to increase the health literacy of the participating men. Beyond that, men's sheds are often accompanied by specific events concerning health-related topics, such as diabetes or Alzheimer's. AMSA, for instance, has designed a specific health program, which aims to encourage older men to participate in prevention programs as well as to raise awareness regarding their individual health by using a gender- and age-specific approach [12]. 
Prior research revealed various health-related effects of community-based men's sheds. Milligan et al. (2013), for example, identified positive impacts on social isolation and psychological well-being of older men participating in men's sheds [13]. Yet, only two reviews are available which both found an insufficient evidence base regarding this topic $[11,13]$. Since the wider implementation of men's sheds is a recent phenomenon, most of the literature on men's sheds has been published after 2013 and therefore was not included in these two reviews. A first non-systematic search also showed that studies assigned to a higher degree of evidence were rather published after 2013. To gain a comprehensive understanding of the current evidence base, a mixed-methods systematic review which includes both quantitative and qualitative studies is required.

\section{Objective and research questions}

The aim of this research project is to strengthen the evidence base of men's sheds by analysing their effectiveness and to gather sufficient information regarding a successful implementation of men's sheds in Germany. It will be guided by the following main research question:

What is the scientific evidence for the impact of the effects of community-based men's sheds on a) self-rated health, b) subjective well-being, and c) social isolation of older men aged 50 years and older in comparison to men who don't participate in men's sheds?

Since there is little practical experience with the implementation of community-based men's sheds in Germany, additional findings regarding a possible transfer of the concept will be included. Therefore, the systematic review also aims to answer the following further research questions:

Which subgroups of older men participate in men's sheds or their individual components and which do not?

What general conditions (e.g. opening hours) exist and what influence do they have on participation? Which potentially undesirable effects are reported and how can they be prevented?

What are the characteristics of a successful men's shed in terms of participation and sustainability? Are they, for instance, equally successful in urban and rural areas?

\section{Methods}

This systematic review protocol follows the reporting guidelines of the Preferred Reporting Items for Systematic Reviews and Meta-Analyses Protocols (PRISMA-P) statement (see checklist in Additional file 1). The systematic review is registered at the International Prospective Register of Systematic Reviews (PROSPERO; registration number forthcoming).

The systematic review will be conducted following the guidelines of The Joanna Briggs Institute (JBI) [14]. The System for the Unified Management, Assessment and Review of Information (SUMARI) - a web application developed by JBI - will be used throughout the whole process [15].

\section{Eligibility criteria}

\section{Study designs}

Both, qualitative and quantitative studies will be included without any restrictions in regard of the study design.

\section{Participants}

The systematic review focuses on older men aged 50 years and above. Therefore, studies will be included if a minimum of $50 \%$ of the study population are at least 50 years old or if separate results for the target population are described.

\section{Interventions}

Studies will be included if they investigate complex community-based interventions that explicitly refer to the concept of community-based men's sheds. A men's shed is a public non-profit communal institution for men where they can engage in joint 
activities. Studies on other community-based activities by men such as voluntary fire brigades in rural areas or men's regulars will not be included.

\section{Comparators}

Men who do not participate in men's sheds serve as the control group.

\section{Outcomes}

The primary outcomes of the systematic review are a) self-rated health, b) subjective well-being, and c) social isolation.

Self-rated health (a) is a frequently used parameter in epidemiology and public health [16]. A typical measuring instrument is a single question from the 36-item Short Form Survey [17]. It has a high content validity and shows a high correlation with morbidity parameters such as the presence of physical complaints or the number of chronic diseases [18].

Subjective well-being (b) is also a very well-established construct of health research. A widely used instrument is the 5-item questionnaire of the World Health Organization [19], which has a high clinical validity [20] and good test-retest reliability [21].

The multidimensional construct of social isolation (c) is measured differently in quantitative studies and has also been investigated under various terms (i.e. "social isolation", "lack of social network", "loneliness") [8]. Therefore, an assessment of validity and reliability must remain exemplary at this point and can only be examined more closely based on the studies included. A frequently used scale is the Lubben Social Network Scale (LSNS), which has been translated into several languages and adapted to cultural needs. The LSNS showed high validity in a 3-country sample of older adults [22].

Secondary outcomes include the characteristics of men who participate in men's sheds and of those who do not, the influence of general conditions of men's sheds on participation, and the characteristics of successful men's sheds in terms of participation and sustainability.

\section{Language}

Studies published in English, German, or French will be included in the review.

\section{Search strategy}

The databases Medline (via PubMed), Scopus, Web of Science, and OpenGrey will be searched for potentially relevant studies. The search term will focus on the intervention. Further restrictions are not necessary as studies on men's sheds are still scarce. The piloted search terms can be found in Additional file 2. There are no restrictions in regard of the publication date.

A search on the websites of the men's sheds associations will be conducted to find further relevant publications. A hand-search in the reference lists of the included publications will complete the search.

\section{Study selection}

Results of the searches will be exported to JBI SUMARI. First, two authors (LF, BMA) will independently screen the studies by titles and abstracts. Discrepancies are solved by consensus procedures or by a third author (KB). Next, full texts of potentially relevant studies will be reviewed by two independent authors (LF, BMA). Any discrepancies are also solved by consensus procedures or by a third author (KB). Inter-rater agreement will be calculated in SUMARI.

\section{Data extraction}

Data of the included studies will be extracted by two authors (LF, BMA) independently. Discrepancies are solved by consensus procedures or by a third person (KB). The standardized JBI tools will be used for data extraction [14]. These will be modified and extended in conjunction with health promotion practitioners. The following information will be extracted: publication details, study design, participants' characteristics, intervention details, details of the control group, and primary and secondary outcomes. Original authors will be contacted if further non-published data is needed.

\section{Quality assessment}


The JBI critical appraisal checklists [14] will be used for the assessment of methodological quality of the selected studies. Quantitative and qualitative studies will be critically assessed with different checklists depending on their study design. All checklists contain several questions to determine whether a study has addressed the risk of bias in its design, conduct, and analyses. These questions must be answered with yes, no, unclear, or not applicable.

Two authors (LF, BMA) will conduct the assessment independently. Discrepancies are solved with involvement of a third author (KB) by consensus procedures. Agreement between reviewers will be assessed for each category by intra-class correlation coefficient.

The methodological quality of the studies will have no influence on their inclusion. Instead, a sensitivity analysis based on the study quality will be conducted to determine the robustness of the results (studies with lower quality vs. studies with higher quality).

\section{Data synthesis}

In our mixed-methods review, we will follow a convergent segregated approach, which involves independent synthesis of quantitative and qualitative data [14]. Subsequent to the separate generation of evidence for each synthesis, the thereby derived results will be integrated.

For quantitative data, a narrative synthesis will be undertaken to summarize the results. However, after preliminary searches we do not expect a meta-analysis to be feasible, since quantitative research appears to be scarce for this topic. If the quantity, heterogeneity, and variation of studies allows it, a meta-analysis will be conducted. If data is available, sub-group analyses by social class, migration status, age group, and health status will be conducted. For the quantitative studies, a funnel plot will be used to detect publication bias.

Qualitative results are combined, where possible, and aggregated by categorizing these findings based on similarity in meaning (meta-aggregation). Where this is not possible, the results are presented in narrative form.

Subsequently, the created data syntheses are combined in a mixed-methods data synthesis. The characteristics of all included studies will be displayed in a tabular summary and the results of the quantitative and qualitative synthesis are transferred into thematic statements and summarized. The development of conclusions and, if possible, recommendations concludes this step.

\section{Assessment of the confidence of evidence}

The confidence of evidence will be assessed according to the recommendations by JBI [23]. These recommendations are based on the Grading of Recommendations Assessment, Development and Evaluation (GRADE) approach, a widely used method to appraise quantitative studies [24]. The JBI tool allows to assess the levels of evidence and grades of recommendation for both quantitative and qualitative studies and was, therefore, chosen for this review. Results of the assessment of the confidence of evidence will be displayed in a 'Summary of Findings' table [25].

\section{Discussion}

The objective of the mixed-methods systematic review is to analyse the effectiveness of men's sheds with regard to self-rated health, subjective well-being, and social isolation. Moreover, we want to gather information on how to successfully implement men's sheds in Germany.

Older men are a rather neglected group in health promotion [5]. Although significant sex differences in regard of life expectancy, morbidity, and health-related behaviour exist [1], there is a lack of men-specific health promotion interventions [6]. The communitybased approach of men's sheds could fill this gap. Over the course of the last years, several studies investigating different aspects of men's sheds have been published (e.g. [26-28]). Synthesizing the results from this wide spectrum of publications will strengthen the evidence of the potential impact of men's sheds on men's health and will moreover summarize important practicerelated information available on this concept. The mixed-methods approach of the systematic review includes both quantitative and qualitative publications and will, therefore, provide a comprehensive overview of the published findings. 
A limitation of the systematic review is that only studies published in English, German, or French will be included. However, we do not expect this to bias results as the concept of men's sheds originated in English-speaking countries, and sheds seem to be most prominent in these countries.

If the results regarding the effectiveness of men's sheds are promising, the review could encourage other countries to consider men's sheds for the promotion of men's health. Furthermore, the results of the review will provide useful implications for the implementation process.

\section{Abbreviations}

AMSA Australian Men's Shed Association

GRADE Grading of Recommendations Assessment, Development and Evaluation

JBI The Joanna Briggs Institute

LSNS Lubben Social Network Scale

PRISMA-P Preferred Reporting Items for Systematic Reviews and Meta-Analyses Protocols

PROSPERO International Prospective Register of Systematic Reviews

SUMARI System for the Unified Management, Assessment and Review of Information

\section{Declarations}

\section{Ethics approval and consent to participate:}

Not applicable.

\section{Consent for publication:}

Not applicable.

\section{Availability of data and materials:}

Not applicable.

\section{Competing interests:}

The authors declare that they have no competing interests.

\section{Funding:}

The MASH project is funded by the German Federal Ministry of Education and Research, (BMBF; grant number 01EL2026). The content of this article reflects only the authors' views and the funder is not liable for any use that may be made of the information contained therein.

\section{Authors' contributions:}

BMA and LF developed the first draft of the protocol. KB supervised the entire manuscript writing and contributed to the revision of the protocol. All authors have read and approved the manuscript.

\section{Acknowledgements:}

Not applicable. 


\section{References}

1. WHO Commission on Social Determinants of Health. Closing the gap in a generation: Health equity through action on the social determinants of health; 2008.

2. Statistisches Bundesamt. Pressemitteilung Nr. 377 vom 29. September 2020. 2020. https://www.destatis.de/DE/Presse/Pressemitteilungen/2020/09/PD20_377_12621.html. Accessed 13 Nov 2020. Accessed 13 Nov 2020.

3. Banks I. No man's land: men, illness, and the NHS. BMJ 2001. doi:10.1136/bmj.323.7320.1058.

4. Carroll P, Kirwan L, Lambe B. Engaging 'hard to reach' men in community based health promotions. Int J Health Promot Educ 2014. doi:10.1080/14635240.2013.876185.

5. Robertson S, Witty K, Zwolinsky S, Day R. Men's health promotion interventions: what have we learned from previous programmes? Community Practitioner. 2013;86:38-41.

6. Doyal L. Sex, gender, and health: the need for a new approach. BMJ 2001. doi:10.1136/bmj.323.7320.1061.

7. Australian Men's Shed Association. About Men's Sheds. 2017. https://mensshed.org/what-is-a-mens-shed/. Accessed 13 Nov 2020.

8. Cudjoe TKM, Roth DL, Szanton SL, Wolff JL, Boyd CM, Thorpe RJ. The Epidemiology of Social Isolation: National Health and Aging Trends Study. J Gerontol B Psychol Sci Soc Sci 2018. doi:10.1093/geronb/gby037.

9. Umberson D, Montez JK. Social Relationships and Health: A Flashpoint for Health Policy. J Health Soc Behav 2010. doi:10.1177/0022146510383501.

10. Milligan C, Payne S, Bingley A, Cockshott Z. Evaluation of the men in sheds pilot programme. London: Age UK; 2012.

11. Wilson NJ, Cordier R. A narrative review of Men's Sheds literature: reducing social isolation and promoting men's health and well-being. Health Soc Care Community 2013. doi:10.1111/hsc.12019.

12. Australian Men's Shed Association. Spanner in the Works? - When was your last service? 2019. https://malehealth.org.au/. Accessed 13 Nov 2020.

13. Milligan C, Dowrick C, Payne S, Hanratty B, Neary D, Irwin P, Richardson D. Men's Sheds and other gendered interventions for older men: improving health and wellbeing through social activity: A systematic review and scoping of the evidence base. UK: Lancaster University Centre for Ageing Research; 2013.

14. Aromataris E, Munn Z. JBI Manual for Evidence Synthesis. 2020. https://synthesismanual.jbi.global. Accessed 13 Nov 2020.

15. The Joanna Briggs Institute. The System for the Unified Management, Assessment and Review of Information (SUMARI). 2020. https://www.jbisumari.org.

16. Jylhä M. What is self-rated health and why does it predict mortality? Towards a unified conceptual model. Soc Sci Med 2009. doi:10.1016/j.socscimed.2009.05.013.

17. Jenkinson C, Wright L, Coulter A. Criterion validity and reliability of the SF-36 in a population sample. Quality of Life Research 1994. doi:10.1007/BF00647843.

18. Baćak V, Ólafsdóttir S. Gender and validity of self-rated health in nineteen European countries. Scand J Public Health 2017. doi:10.1177/1403494817717405.

19. Bech P, Gudex C, Johansen KS. The WHO (Ten) Well-Being Index: Validation in Diabetes. Psychother Psychosom 1996. doi:10.1159/000289073.

20. Topp CW, Østergaard SD, Søndergaard S, Bech P. The WHO-5 Well-Being Index: A Systematic Review of the Literature. Psychother Psychosom 2015. doi:10.1159/000376585.

21. Schougaard LMV, Thurah A de, Bech P, Hjollund NH, Christiansen DH. Test-retest reliability and measurement error of the Danish WHO-5 Well-being Index in outpatients with epilepsy. Health Qual Life Outcomes 2018. doi:10.1186/s12955-018-10010 .

22. Blozik E, Wagner JT, Gillmann G, lliffe S, Renteln-Kruse W von, Lubben J, et al. Social network assessment in communitydwelling older persons: results from a study of three European populations. Aging Clin Exp Res 2009.

doi:10.1007/BF03325223. 
23. The Joanna Briggs Institute. Supporting Document for the Joanna Briggs Institute Levels of Evidence and Grades of Recommendation. 2014. https://joannabriggs.org/sites/default/files/2019-

05/JBI\%20Levels\%20of\%20Evidence\%20Supporting\%20Documents-v2.pdf. Accessed 20 Nov 2020.

24. Guyatt GH, Oxman AD, Vist GE, Kunz R, Falck-Ytter Y, Alonso-Coello P, Schünemann HJ. GRADE: an emerging consensus on rating quality of evidence and strength of recommendations. BMJ 2008. doi:10.1136/bmj.39489.470347.AD.

25. The Joanna Briggs Institute. Summary of Findings Tables for Joanna Briggs Institute Systematic Reviews. 2016. https://nursing.Isuhsc.edu/JBI/docs/Grade/Summary_of_Findings_Tables_for_Joanna_Briggs_Institute_Systematic_ReviewsV3.pdf. Accessed 20 Nov 2020.

26. Nurmi MA, Mackenzie CS, Roger K, Reynolds K, Urquhart J. Older men's perceptions of the need for and access to malefocused community programmes such as Men's Sheds. Ageing \& Society 2018. doi:10.1017/S0144686X16001331.

27. Waling A, Fildes D. 'Don't fix what ain't broke': evaluating the effectiveness of a Men's Shed in inner-regional Australia. Health Soc Care Community 2017. doi:10.1111/hsc.12365.

28. Ormsby J, Stanley M, Jaworski K. Older men's participation in community-based men's sheds programmes. Health Soc Care Community 2010. doi:10.1111/j.1365-2524.2010.00932.x.

\section{Supplementary Files}

This is a list of supplementary files associated with this preprint. Click to download.

- Additionalfile1.docx

- Additionalfile2.docx 